\title{
Recurrent cerebral abscesses in a child with hepato-pulmonary syndrome
}

*Nimesha Chamidani Gamhewage ${ }^{1}$, Jithangi Wanigasinghe ${ }^{2}$, Pratheep Navabalasooriya ${ }^{1}$, Shehan Perera ${ }^{1}$, Nadeesha Weerasinghe ${ }^{1}$, V P Wickramasinghe ${ }^{2}$, Harendra de Silva ${ }^{2}$

Sri Lanka Journal of Child Health, 2018; 47(2): 166-168

DOI: http://dx.doi.org/10.4038/sljch.v47i2.8485

(Key words: Chronic liver disease, hepato-pulmonary syndrome, cerebral abscess)

\section{Introduction}

Hepato-pulmonary syndrome (HPS) is characterised by the development of pulmonary precapillary and capillary dilatation, pleural and pulmonary arteriovenous shunts and porto-pulmonary anastomoses. This is a known complication in chronic liver disease (CLD) and is reported in 9 to $20 \%$ of children with cirrhosis 1 .

\section{Case report}

An eleven year old girl who has undergone Rouxen-Y portoenterostomy (Kasai procedure) at six weeks of age for biliary atresia, presented with fever, severe headache and vomiting of four days duration. She had satisfactory growth and

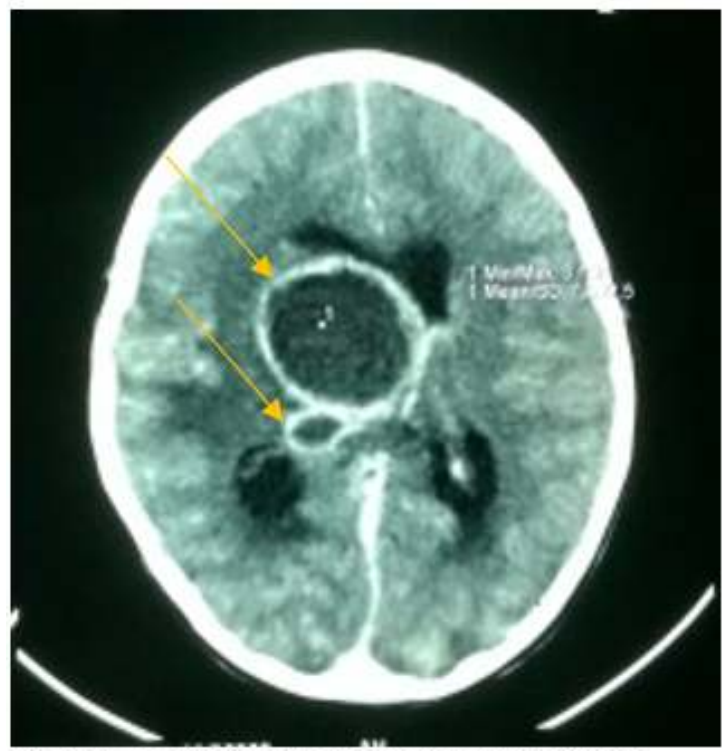

Figure 1: CT brain of previous admission showing multiple abscesses (shown by arrows)

${ }^{1}$ Lady Ridgeway Hospital for Children, Colombo, Sri Lanka, ${ }^{2}$ Faculty of Medicine, Colombo, Sri Lanka

*Correspondence:nimeshagamhewage@gmail.com (Received on 20 October 2016: Accepted after revision on 18 November 2016)

The authors declare that there are no conflicts of interest

Personal funding was used for the project.

Open Access Article published under the Creative

Commons Attribution CC-BY

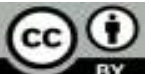

development until the age of 8 years when she had presented with similar symptoms and was found to have multiple abscesses in the right fronto-parietal and thalamic regions (Figure 1). The abscesses were managed with aspiration and intravenous antibiotics. She suffered from residual left sided hemiparesis which did not recover completely due to defaulted rehabilitation.

During the current admission she was febrile, drowsy and ill looking with signs of meningism. She was plethoric and cyanosed with finger clubbing. There was no jaundice, palmar erythema, spider naevi or oedema. Liver size was normal but splenomegaly of $5 \mathrm{~cm}$ was noted. Neither ascites nor pulmonary hypertension was noted. Heart was clinically normal. There was new onset right sided hemiparesis with residual left sided cogwheel rigidity and intention tremors from the previous cerebral insult. Her blood counts showed a leucocytosis of $24,000 / \mathrm{cu} \mathrm{mm}$ with $80 \%$ neutrophils, a haemoglobin of $17.2 \mathrm{~g} / \mathrm{dl}$, a platelet count of $93,000 / \mathrm{cu} \mathrm{mm}$ and a C-reactive protein (CRP) of $267 \mathrm{mg} / \mathrm{dl}$. The magnetic resonance imaging (MRI) of brain revealed multiple abscesses; in the left fronto-parietal and periventricular regions. (Figure 2).

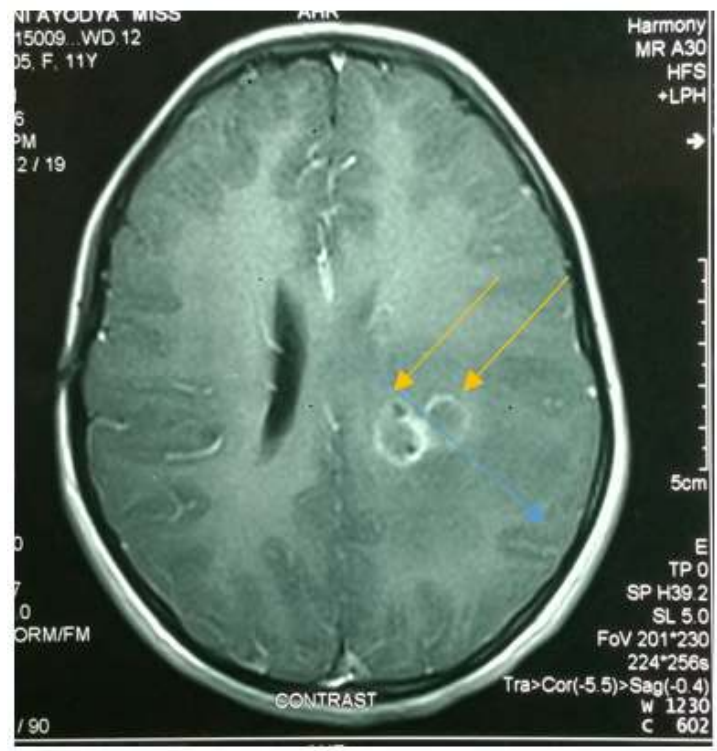

Figure 2: MRI of brain during current admission showing multiple abscesses (shown by arrows) 
Liver function tests showed normal synthetic functions, elevated serum transaminases and mild conjugated hyperbilirubinaemia. Serum alkaline phosphatase was elevated. Renal functions were normal. Hypoxaemia was demonstrated in the arterial blood gas analysis. Ultrasound scan of abdomen showed increased echogenicity of liver parenchyma with moderate splenomegaly. Upper gastrointestinal endoscopy did not reveal oesophageal varices. Her echocardiogram showed a structurally normal heart. Bubble contrast study (Figure 3) with agitated saline showed late appearance of injected bubbles to left atrium after the third cardiac cycle but before the fifth indicating shunting within the pulmonary system.

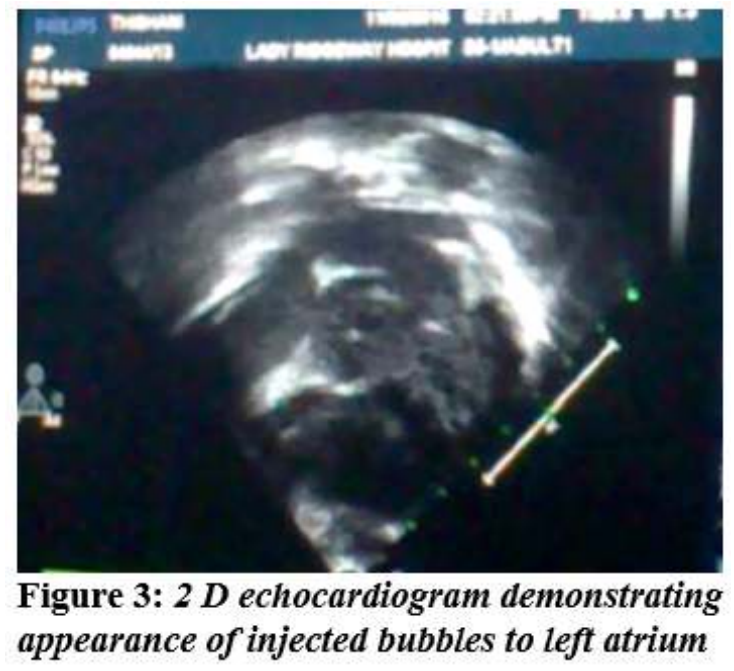

Pulmonary angiogram revealed diffuse bilateral pulmonary arteriovenous malformations (Figure 4).

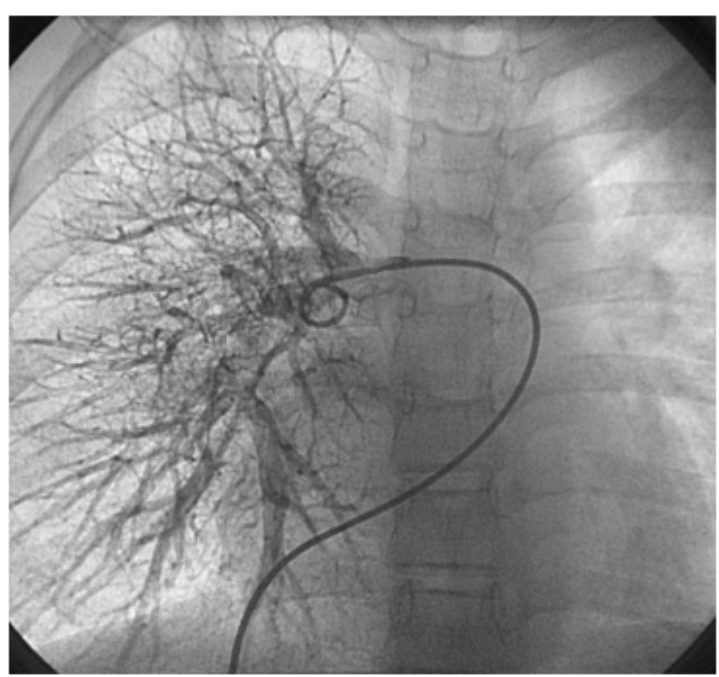

Figure 4: Diffuse arteriovenous malformations

She underwent aspiration of the cerebral abscesses followed by intravenous antibiotics for six weeks. Remarkable recovery of right sided weakness was observed with intensive rehabilitation. Improvement of her extrapyramidal signs and left sided weakness was less impressive.

\section{Discussion}

In patients with CLD, two types of pulmonary vascular syndromes are recognised. They are: portopulmonary hypertension and $\mathrm{HPS}^{1}$. HPS is characterised by the combination of CLD, arterial hypoxaemia and intrapulmonary vascular dilatation $^{3}$. Presence of portal hypertension is not a prerequisite. Furthermore, there is no correlation between the severity of HPS and the degree of $\mathrm{CLD}^{1}$. Clinical manifestations include cyanosis, digital clubbing, platypnoea, dyspnoea and orthodeoxia ${ }^{4}$. Vasodilation of pulmonary capillaries causes alveolar ventilation perfusion mismatch. Pleural and pulmonary arteriovenous shunts lead to rapid exit of poorly oxygenated blood into pulmonary veins ${ }^{1}$. Right to left shunting reduces the filtering capacity of organisms by the lung predisposing to brain abscess formation during transient bacteraemia ${ }^{5}$.

Multiple mechanisms have been postulated to cause intrapulmonary vasodilation. These include failure to clear circulating pulmonary vasodilators, production of circulating vasodilators and inhibition of a circulating vasoconstrictive substance by the damaged liver. Nitric oxide (NO), bowel endotoxins, TNF-alfa and prostaglandins are some of the proposed mediators ${ }^{1}$.

Diagnosis of HPS is established by demonstrating pulmonary vessel dilation and arteriovenous communications either by two dimensional contrast echocardiography or macro aggregated albumin lung perfusion $\operatorname{scan}^{3}$. For the first, agitated saline is used as a contrast medium. After intravenous administration, it creates a stream of microbubbles. Dilated capillaries in HPS allows these microbubbles to enter into left atrium within 3-5 cycles. The distinction between intrapulmonary or intracardiac shunt is made by the time of appearance of the microbubbles in the left heart chambers: in intracardiac shunt the microbubbles appear generally within three cardiac cycles. In intrapulmonary vascular dilations bubbles appear in left atrium after three cardiac cycles ${ }^{1}$.

In adults, prognosis is poor without liver transplantation. Data regarding outcome in children is lacking. Shunting and hypoxaemia can be reversed by liver transplantation ${ }^{6}$. Medical management with inhibition of nitric oxide and methylene blue has been attempted. However no long term benefits have been observed ${ }^{1}$. Embolization of large pulmonary arteriovenous fistulas is done as a bridging strategy awaiting liver transplantation. In our patient who had bilateral 
malformations, liver transplantation remained the option of management.

More and more children with biliary atresia who have undergone Kasai procedure survive till adolescence. Therefore it is important to routinely screen patients for the presence of hepatopulmonary syndrome and direct for transplantation appropriately. The transplantation procedure is not available in Sri Lanka for patients like ours as well as those who fail the Kasai procedure. It is prohibitively expensive in other countries. Therefore, we would like to emphasise the need for establishment of a liver transplant programme for children in Sri Lanka.

\section{References}

1. Deep A, Jose B, Dhawan A. Hepatopulmonary syndrome in children-an update. Paediatrics and Child Health. 2015; 25(6):282-5.

https://doi.org/10.1016/j.paed.2015.02.002

2. Morita K, Fukuzawa H, Maeda K. Brain abscess in hepato-pulmonary syndrome associated with biliary atresia. Pediatrics International 2015; 57: 1187-9.

https://doi.org/10.1111/ped.12711

PMid: 26711920
3. Schenk P, Fuhrmann V, Madl C, Funk G, Lehr S, Kandel O, Müller C. Hepatopulmonary syndrome: prevalence and predictive value of various cut offs for arterial oxygenation and their clinical consequences. Gut 2002; 51(6):853-9. https://doi.org/10.1136/gut.51.6.853 PMid: 12427789 PMCid: PMC1773478

4. Lange PA, Stoller JK. The hepatopulmonary syndrome. Annals of Internal Medicine 1995; 122(7):521-9. https://doi.org/10.7326/0003-4819-122-7199504010-00008

5. Molleston JP, Kaufman BA, Cohen A, Shackelford PG, Keating JP, Lowell JA, Howard TK. Brain abscess in hepatopulmonary syndrome. Journal of Pediatric Gastroenterology and Nutrition 1999; 29(2): 225-6.

https://doi.org/10.1097/000051761999080 00-00024

PMid: 10435665

6. Lange PA, Stoller JK. The hepatopulmonary syndrome: effect of liver transplantation. Clinics in Chest Medicine 1996; 17(1):115-23.

https://doi.org/10.1016/S02725231(05)70 302-6 